\title{
Can Your Smartphone Detect Your Emotion?
}

\author{
Daxiang Dai \\ Chongqing Key Laboratory of \\ Computational Intelligence \\ Chongqing, China
}

\author{
Qun Liu \\ Chongqing Key Laboratory of \\ Computational Intelligence \\ Chongqing, China
}

\author{
Hongying Meng \\ Electronic and Computer Engineering \\ Department \\ Brunel University, UK
}

\begin{abstract}
The smartphone has become an indispensable part in people's life. Identifying the user's emotional state according to the usage of smartphone is a new way to improve the humancomputer interaction and user experience. In this paper, we present an attempt to recognize emotional states by using fingerstroke pattern. Firstly, International Affective Picture System (IAPS) were used to design the emotion inducing experiment. Then finger-stroke features under different emotional categories were extracted and analyzed. Ultimately, we used machine learning algorithms to identify three basic emotional states including positive, neutral, and negative. The experiment results show that the stroke exists some specific behavioral patterns between different people. For all 24 subjects, the average classification accuracy rate reached $85.1 \%$, and we got the average recognition accuracy at $72.3 \%, 74.6 \%$ and $69.6 \%$ for male, female and all subjects.
\end{abstract}

Keyword-Smartphone; Emotion recognition; Human-computer interaction; Machine learning.

\section{Introduction}

With the coming of the information era, the smartphone has become the essential part in most people's life. In 2014, Google published The Consumer Barometer pointing out that the rate of smartphone population is highest in Singapore with $85 \%$ and Korea with $80 \%$, but $66 \%$ in China and $53 \%$ in America. With the continuous development of information technology, the intelligent device will be more and more popular, but brings more challenges as well. For instance, the user experience can't be content with the individual demands among different groups. Emotion plays a very important role in people' life, which effects people' judgement, decision and other behaviors. If the smartphone can recognize the users' emotion state automatically, it will make a great improvement of the Humancomputer interaction, individual recommend and other user experience.

The study of psychology indicates that touch behavior as one of nonverbal communicative modes can also reflect a person's emotion condition just like facial expression and body language. Matthew J. Hertenstein, et al. claimed that humans can communicate numerous emotions such as anger, fear, disgust, love, gratitude, and sympathy via touch behavior[1][2]. Erin H. Thompson, et al. compared strangers and couples to communicate emotions solely via touch. Results showed that both couples and strangers were able to communicate prosocial emotions such as love, anger, fear and so on [3].

In recent years, emotion recognizing with touch gesture is widely used in artificial intelligence fields like robots and so on. Steve, et al. examine how humans communicate emotional state through touch to the Haptic Creature, a touch-centric social robot to further analyze the human's higher intent in communication[4]. Anna, et al. applied affective touch-sensing zoomorphic prototype with pressure and conductive fur sensors to recognize 9 key affective touch gestures and then deduced people's emotion condition [5].

This paper presents a method to identify user's emotion by extracting the feature of stroking screen when people use of smartphone. As we know, the smartphone are equipped with a touch screen and a variety of sensors. When people stroke the screen, sensors will record the user's finger sliding data including coordinate, time, speed and pressure for every stroke.

This work is supported by ChongQing postgraduate scientific research and innovation projects of China, partly funded by the National Nature Science Foundation of China (61379114) and the Natural Science Foundation of Chongqing (CSTC2014jcyjA40047) and the Doctoral startup project of CQUPT (A2014-20). 
Extracting features from these data and using machine learning classification algorithm to train the classifier, thus achieving the purpose of identifying the user's emotional state through the smartphone.

This paper is organized as follows: Section 2 introduces the related work about emotion recognition. Section 3 to Section 5 refer to the major methods of research, and Section 3 introduces the way of inducing emotion, Section 4 introduces the procedure of collecting data and label, Section5 introduces the method of emotional recognition. Section 6 is the result of the experiment and some related analysis. Section 7 summarizes this paper and proposes the next phase of research work.

\section{Related work}

Emotion is a series of mental state reflected by the attitude of the subject to the objective things. Emotion influences every aspect of our lives and plays an important role in people's decision-making and communication. Thus, the emotion recognition technology has very important practical significance and caused wide public concern in various research fields over the recent years.

In the field of natural language processing (NLP), Cheng, et al. proposed a framework to estimate the sentiment polarity of reviews by virtue of opinion and lexica and key sentences extracted from unlabeled data[6][7]. First extracting the candidate opinion words by the adverb like 'very' and 'highly'. Then a key sentence extraction method is developed based on the extracted opinion lexicon. Last a sentiment classifier is generated by self-supervised learning (SSL) method. Microblog has been widely studied as a highly influential social tool. Liu, et al. presented a microblogging emotion recognition method by extracting textual features and non-textual features[8][9]. Traditional sentiment textual features include a variety of key words to reflect the emotional state and nontextual features include the common emoticons, temporal features and punctuation in micro-blog.

Emotional changes are accompanied by a series of physiological responses. By extracting the characteristics of the physiological signals, it can also identify people's emotional state. Jang, et al. measured physiological electrodes for acquisition of bio-signals such as ECG, EDA, PPG and SKT were placed on their bilateral wrists, fingers, and ankle. By extracting these bio-signal features to identify emotions [10]. Zhang, et al. presented an innovative emotion recognition approach which combines EEG signals and pupillary response collected from eye tracker [11]. By extracting the feature of EEG signal and eye tracker under different emotion, the model of emotion is built by using support vector machine, which can efficiently recognize and categorize user's emotion.

Traditional emotion recognition technologies include facial expression recognition and speech emotion recognition. With the development of information technology recently, the research to identify the user's emotion by using of contact with some external devices access to a wide range of study. Khanna, et al. presented a method to recognize selected emotion categories from keyboard stroke pattern, thus verify the exist significant difference between typing speed, the frequency of using backspaces and use of unrelated keys[12]. Touch behavior can not only play a role in social communication, but also can reflect person's emotional state to a large extent. The pressure sensor and position sensor carried by the bionic robot are used to capture people's touching behavior. Then the machine learning methods are used to analyze people's emotion and intention. Anna et al. used a furry social robot to detect and recognize stroke, scratch and light touches. Based on conductive fur, they demonstrated a new type of sensor, which captures motion by measuring changing current as the fur's conductive threads connect and disconnect during touch interaction [13]. Jung et al. extracted the features of the change of position and pressure by the sensor wrapped around the mannequin arm, based on which gesture recognition and emotion analysis are conducted [14].

In recent years, the research of recognizing the user's emotional state through the smartphone has also been studied by some scholars. Gao et al. designed a game named Samurai Fruit to capture players' touch behavior, and then classified emotion with machine learning algorithms [15]. Gerald, et al. proposed a method to detect stress-related changes in the behavior of individuals by using smartphones [16].

In this paper, we trained classifiers for single tester, different gender and all testers respectively. Firstly, the emotion induct experiment was designed to guide testers' basic 
emotional states including positive, neutral and negative, then we extracted behavior characteristics of stroking screen under different emotions. At last, we labelled the feature data and trained the emotion recognizing classifiers with classification algorithm of machine learning. Eventually, we realized our purpose, recognizing the emotion states by using the smartphone.

\section{Emotion induction}

In the domain of psychology, researchers mostly use the affective pictures, music, or movie clips to induce specific emotional states. In this paper, we use International Affective Picture System (IAPS) and E-prime which is a psychological software to design emotional induction experiment. By this way, we can induct three basic emotional states including positive, neutral and negative for every testers. Fig.1. show an example of the IAPS used in the experiment.

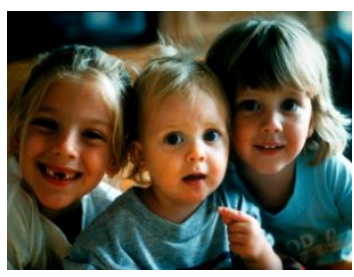

(a) Positive

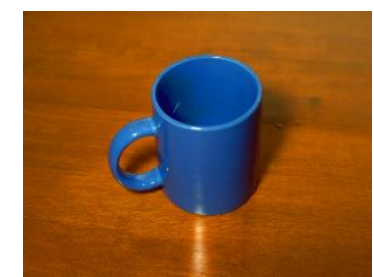

(b) Neutral

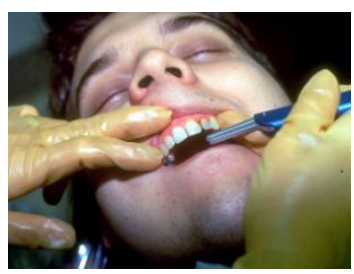

(c) Negative

Fig. 1. An example of three kinds of emotional state in IAPS.

\section{A. Materials}

Because everyone's perception of affective pictures more or less exist difference and the same picture can express different emotion information for different objects. The discrepancy is more pronounced between different genders, so we set up emotional stimulus picture library for both men and women. We first design a questionnaire, which require 100 volunteers including 60 male and 40 female tags and ratings to the picture of IAPS. Tag is qualitative to affective images, which including positive, neutral and negative. Rating is grade pleasure and arousal to pictures. According to the results of tags and ratings, 120 pictures which have best emotion-inducted effectiveness and intensity were selected to build two group of picture libraries for both men and women.

\section{B. Subjects}

24 college students and graduate students participated in the experiment (12 male and 12 female, 21 25 years). All of them had normal vision or corrected-to-normal vision. All of participants were right-handed, good health, having no previous history of neurological or psychiatric diseases.

\section{Procedure}

Firstly, a fixation presented in the central of computer last $500 \mathrm{~ms}$. After that a positive picture will appear randomly and non-repeatedly last $1000 \mathrm{~ms}$. Then fixations and positive pictures will appear circularly until the group of experiment ended. Due to the induced emotion has timeliness, the emotion which is inducted will decrease with time. Therefore, we divided a group of emotion induction experiments into two parts. When the first part of the emotional induction is completed, we collected the data of stroke. After the induced emotion subsided, we turn into the second part of emotion induction experiment and collection the data of stroke screen. Thus, we will achieve the purpose of getting more effective data by this method. Taking the positive emotion as an example, the experimental process is shown in Fig.2.

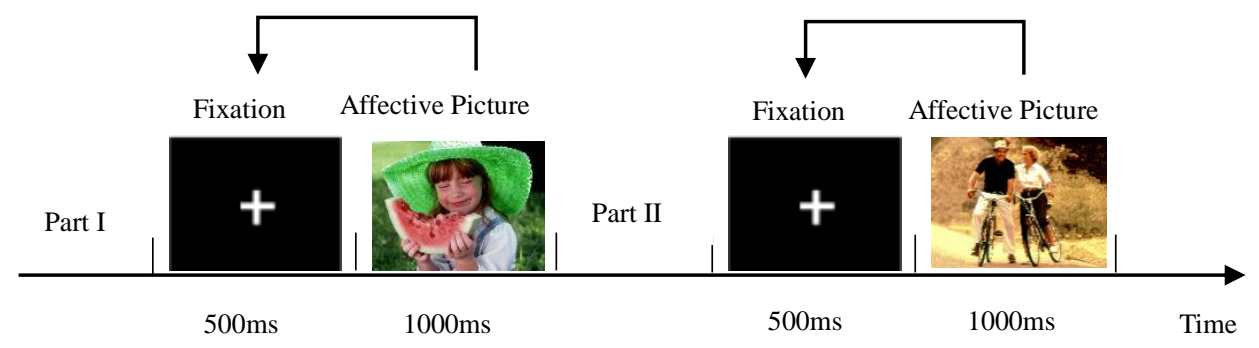

Fig. 2. Time sequence of a group emotion induction. 
Table 1. The data collected and labeled

\section{Data collection and labelling}

\section{A. Data collection}

Smartphones are equipped with a variety of sensors such as touch panel, accelerometer and gyroscope, they can monitor users' touch behavior and sensory data. So we through the platform of Xshell and SSHDroid to design experiments to obtain logs when users stroke the screen of smartphone. Then we can get the relevant data we need.

After the completion of the emotional induction, testers will asked to stroke the screen of smartphone. The system can monitor and record the time, coordinate position and pressure of each slide. As shown in Fig.3. Each stroke records the several time points' information of coordinates and pressure and so on, we take the information of each stroke as a record. The length, time, speed and pressure of each stroke can be obtained through the analysis and processing of these data.

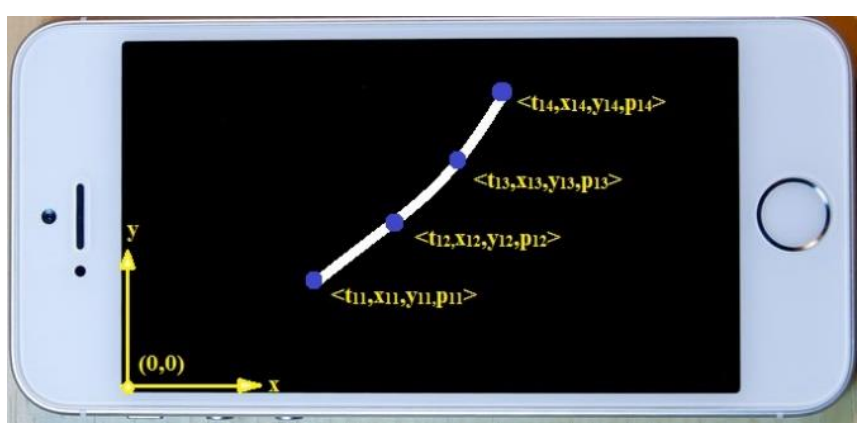

Fig. 3. Coordinates of the points for one stroke.

\section{B. Labelling}

In Section 3, we induce three basic emotions of positive, neutral and negative by the emotion induct experiment. Then we asked 24 testers operation on smartphone and the log will record the data of each stroke under three kinds of emotional labels. In order to get data we need, sometimes we inevitably click to the screen. Therefore, it is necessary to preprocess the data to remove the outliers and abnormal data caused by accidental touch. The tag data we get is shown in Table 1.

24 subjects are experimented and 23100 sets of stroke screen data are obtained by analysis and processing, including 11350 male data and 11750 female data.

\begin{tabular}{|c|c|c|c|c|c|c|c|c|c|}
\hline Gender & No. & Pos. & Neu. & Neg. & Gender & No. & Pos. & Neu. & Neg. \\
\hline \multirow{5}{*}{ Male } & 1 & 240 & 250 & 300 & \multirow{5}{*}{ Female } & 1 & 300 & 350 & 300 \\
\hline & 2 & 260 & 250 & 300 & & 2 & 275 & 350 & 300 \\
\hline & $\ldots$ & $\ldots$ & $\ldots$ & $\ldots$ & & $\ldots$ & $\ldots$ & $\ldots$ & $\ldots$ \\
\hline & 12 & 350 & 350 & 300 & & 12 & 300 & 300 & 400 \\
\hline & Total & \multicolumn{3}{|c|}{11350} & & Total & \multicolumn{3}{|c|}{11750} \\
\hline Total & \multicolumn{9}{|c|}{23100} \\
\hline
\end{tabular}

\section{Emotion recognition}

\section{A. Feature extraction}

In section 4, we get the length, time, speed and pressure of each stroke. If we extracted features for each stroke record, the result is occasionally. Therefore, we take a continuous 5 records as a segmentation interval, the average, median, maximum, minimum and variance of the length, time, speed and pressure in each segment are extracted. At last, we extracted 20 features.

In order to get the above characteristics, we need to obtain the length, time, speed and pressure of each stroke at first. Their definitions as follows.

Stroke Length: The distance of finger movement from start to end when we stroke the screen of smartphone. The formula for calculation is as follows:

$$
d_{i}=\sum_{j=1}^{n_{i}-1} \sqrt{\left(x_{i(j+1)}-x_{i j}\right)^{2}+\left(y_{i(j+1)}-y_{i j}\right)^{2}}
$$

Stroke Time: The time spent in finger movement from start to end when we stroke the screen of smartphone. The formula for calculation is as follows:

$$
t_{i}=T_{n_{i}}-T_{1 i}
$$

Stroke Speed: The velocity of finger movement from start to end when we stroke the screen of smartphone. The formula for calculation is as follows: 


$$
v_{i}=\frac{d_{i}}{t_{i}}
$$

Stroke Pressure: The average pressure of stroke when we stroke the screen of smartphone. The formula for calculation is as follows:

$$
p_{i}=\frac{\sum_{j=1}^{n_{i}} P_{i j}}{n_{i}}
$$

In formula (1) to formula (4), $d_{i}$ denotes the length for the ith stroke. $n_{i}$ denotes the number of points for ith stroke. $x_{i j}$ and $y_{i j}$ is the $\mathrm{x}$-coordinate and $\mathrm{y}$-coordinate for the jth point of ith stroke. $t_{i}$ denotes the time spent in ith stroke. $T_{n_{i}}$ denotes the time at the end of ith stroke. $T_{1 i}$ denotes the time at the begin of ith stroke. $v_{i}$ denotes the velocity of ith stroke. $p_{i}$ denotes the pressure of ith stroke and $P_{i j}$ is the pressure for the jth point of ith stroke.

We extracted the average, median, maximum, minimum and variance of length, time, speed and pressure as features. Eventually, 20 features are obtained as shown in Table2.

\begin{tabular}{|c|c|c|}
\hline Index & Features & Function \\
\hline \multirow{5}{*}{$\begin{array}{l}\text { Stroke } \\
\text { Length }\end{array}$} & Average & $\bar{d}_{i}=\frac{\sum_{i=1}^{N} d_{i}}{N}$ \\
\hline & Median & $d_{\text {med }}=\operatorname{median}\left(d_{i}\right)$ \\
\hline & Max & $d_{\max }=\max \left(d_{i}\right)$ \\
\hline & Min & $d_{\min }=\min \left(d_{i}\right)$ \\
\hline & Variance & $s^{2}=\frac{1}{N}\left[\left(d_{1}-\bar{d}\right)^{2}+\left(d_{2}-\bar{d}\right)^{2}+\cdots+\left(d_{N}-\bar{d}\right)^{2}\right]$ \\
\hline $\begin{array}{l}\text { Stroke } \\
\text { Time }\end{array}$ & Average & $-\sum_{t=1}^{N} t_{i}$ \\
\hline
\end{tabular}

Table 2. Stroke Features.

\begin{tabular}{|c|c|c|}
\hline & Median & $t_{\text {med }}=\operatorname{median}\left(t_{i}\right)$ \\
\hline & Max & $t_{\max }=\max \left(t_{i}\right)$ \\
\hline & Min & $t_{\min }=\min \left(t_{i}\right)$ \\
\hline & Variance & $s^{2}=\frac{1}{N}\left[\left(t_{1}-t\right)^{2}+\left(t_{2}-t\right)^{2}+\cdots+\left(t_{N}-t\right)^{2}\right]$ \\
\hline \multirow{5}{*}{ Speed } & Average & $-\sum_{i=1}^{N} v_{i}$ \\
\hline & Median & $v_{\text {med }}=$ median $\left(v_{i}\right)$ \\
\hline & Max & $v_{\max }=\max \left(v_{i}\right)$ \\
\hline & Min & $v_{\min }=\min \left(v_{i}\right)$ \\
\hline & Variance & $s^{2}=\frac{1}{N}\left[\left(v_{1}-\bar{v}\right)^{2}+\left(v_{2}-\bar{v}\right)^{2}+\cdots+\left(v_{N}-\bar{v}\right)^{2}\right]$ \\
\hline \multirow{5}{*}{ Stroke } & Average & $\bar{p}=\frac{\sum_{i=1}^{N} p_{i}}{N}$ \\
\hline & Median & $p_{\text {med }}=\operatorname{median}\left(p_{i}\right)$ \\
\hline & Max & $p_{\max }=\max \left(p_{i}\right)$ \\
\hline & Min & $p_{\min }=\min \left(p_{i}\right)$ \\
\hline & Variance & $s^{2}=\frac{1}{N}\left[\left(p_{1}-\bar{p}\right)^{2}+\left(p_{2}-\bar{p}\right)^{2}+\cdots+\left(p_{N}-\bar{p}\right)^{2}\right]$ \\
\hline
\end{tabular}

In Table 2, $N$ is the number of stroke in each segmentation interval. In this paper, $N=5$, for all $i=1,2, \cdots, N$.

\section{B. Feature analysis}

In order to further explore the discrepancy of features between different gender and emotional state, we analyzed the length, time, velocity and pressure of each stoke for all subjects under different emotional conditions. 


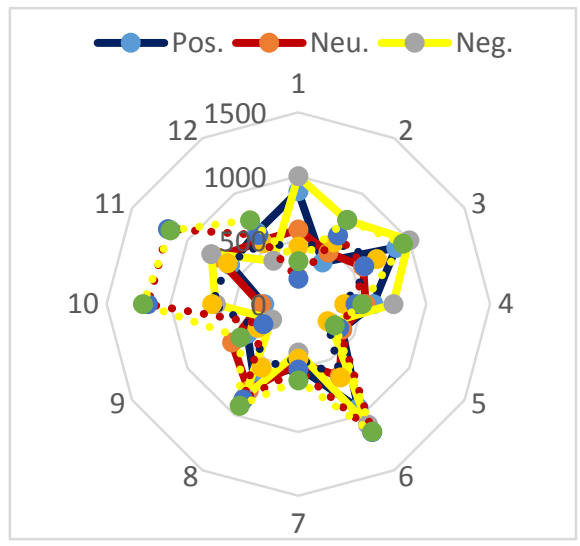

a. Length

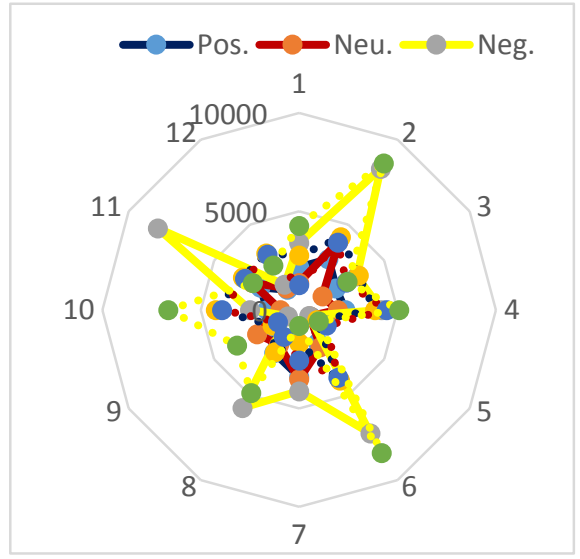

c. Velocity

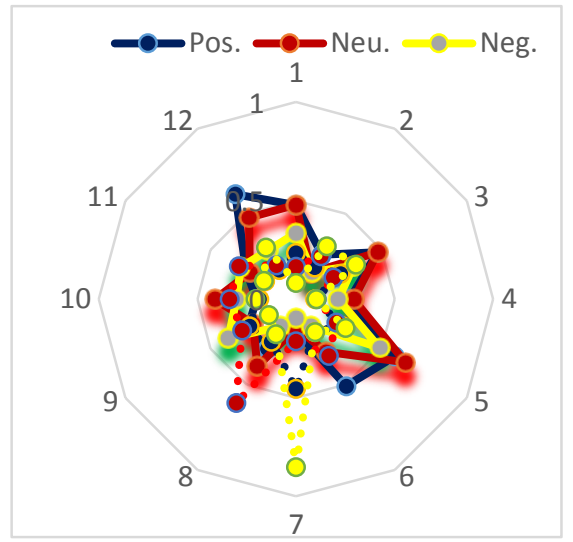

b. Time

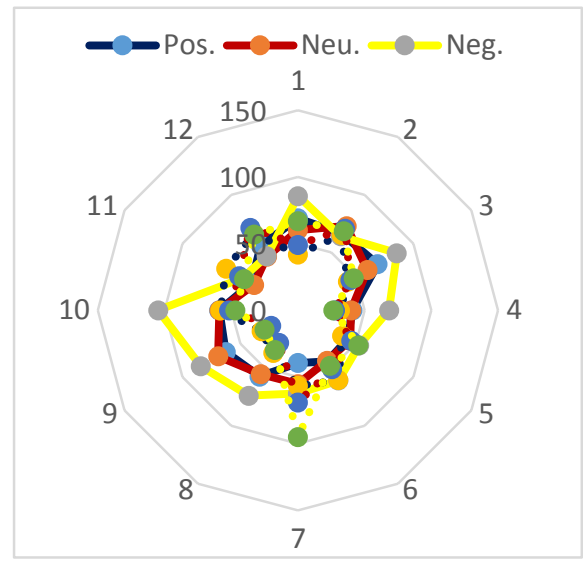

d. Pressure

Fig.4. Radar Chart showing the variation in length, time, velocity and pressure for 24 participants over the 3 basic emotional states.

As shown in Fig.4, in the radar chart, the number 1 to 12 in the outer ring represent 12 subjects. The inner ring to the outer ring represents the increases of the feature in turn. The solid line represents the feature change of male and the dotted line represents the feature change of female. Blue represents positive emotion, Red represents neutral emotion and yellow represents negative emotion. From the picture, we can see that the characteristics of stroke screen are similar under positive and neutral. The speed and pressure under negative emotion are significantly higher than positive and neutral. Besides, the speed and pressure of the male is higher than the female. The time is longer and length is shorter under the emotion of positive and neutral. From this it is clear that the emotional effects induct by positive picture and neutral picture are similar, and it is significant different to the negative. Predictably, people have a stronger reaction to the negative emotion.

\section{Classifier}

The features from Section 5.1 were used for classification using WEKA which is an open source software of machine learning and data mining based on Java. We test a variety of classification learning algorithms by WEKA soft, which contains BayesNet (BN), Back-Propagation neural network (BPNN) and RandomForest (RF). The 10-fold cross-validation was used to test the classification performance of single tester, different gender and all testers.

\section{Experiment results}

\section{A. Personal}

In this section, we will using the classification algorithms of BN, BPNN and RF to train classifiers for 24 subjects. By this way to identify the emotion of positive, neural and negative, the recognition results are shown in Table 3. 
Table 3. Classification results for 24 participants (unit: \%)

\begin{tabular}{|c|c|c|c|c|c|c|c|c|c|}
\hline Gender & No & BN & BP & $\mathrm{RF}$ & Gender & No & BN & BP & $\mathrm{RF}$ \\
\hline \multirow{13}{*}{ Male } & 1 & 88.3 & 87.7 & 86.4 & \multirow{13}{*}{ Female } & 1 & 93.6 & 94.0 & 93.2 \\
\hline & 2 & 88.0 & 84.3 & 89.8 & & 2 & 76.2 & 76.2 & 75.5 \\
\hline & 3 & 91.1 & 91.7 & 92.9 & & 3 & 94.5 & 98.5 & 97.5 \\
\hline & 4 & 70.1 & 70.5 & 74.2 & & 4 & 64.7 & 62.9 & 62.2 \\
\hline & 5 & 77.8 & 73.1 & 74.3 & & 5 & 90.9 & 94.0 & 95.3 \\
\hline & 6 & 96.2 & 95.4 & 96.2 & & 6 & 83.0 & 83.9 & 83.9 \\
\hline & 7 & 83.2 & 86.9 & 84.9 & & 7 & 86.4 & 88.0 & 85.4 \\
\hline & 8 & 85.8 & 81.6 & 87.3 & & 8 & 92.8 & 91.2 & 92.4 \\
\hline & 9 & 75.8 & 76.6 & 74.0 & & 9 & 90.3 & 88.2 & 89.9 \\
\hline & 10 & 89.1 & 87.5 & 88.7 & & 10 & 83.5 & 82.5 & 85.9 \\
\hline & 11 & 83.2 & 85.0 & 88.2 & & 11 & 83.2 & 79.6 & 81.7 \\
\hline & 12 & 94.4 & 95.8 & 95.1 & & 12 & 79.4 & 75.1 & 75.7 \\
\hline & Mean & 85.3 & 84.7 & 86.0 & & Mean & 84.9 & 84.5 & 84.9 \\
\hline Mean & \multicolumn{9}{|c|}{85.1} \\
\hline
\end{tabular}

The results show that the accuracy of emotion recognition from $62.2 \%$ to $98.5 \%$, average rate of recognition is $85.1 \%$. The recognition accuracy of RF classifier algorithm is the highest, for male accuracy rate is $86.0 \%$ and for female is $84.9 \%$. RF algorithm is a kind of multi-classifiers algorithm composed of multiple classification and regression tree. In generally, RF algorithm can balance error and has higher accuracy compared with the traditional single classifier.

\section{B. General}

As we know, the characteristics of stroke screen were different for men and women. So we trained classifiers for men and women respectively. And then we add the gender as a new feature to train the classifier for all subjects based on the original 20 features. The results are shown in Fig.5.

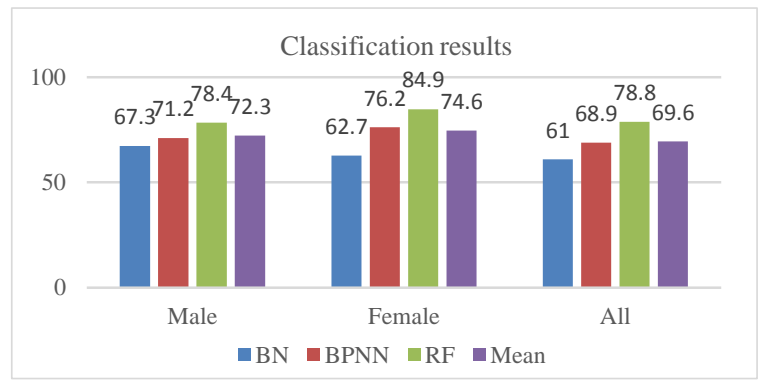

Fig. 5. Classification results for different genders and all testers (unit: \%).
The results show that the average recognition rate reach $72.3 \%$ for male, $74.6 \%$ for female and $69.6 \%$ for all subjects. The recognition effect of RF algorithm is better than $\mathrm{BN}$ and BPNN, and it is reach $78.4 \%, 84.9 \%$ and $78.8 \%$ for male, female and all subjects by using RF algorithm.

We take the RF algorithm as an example, to further analysis the classification results for all subjects. We get the confusion matrix as shown in Fig.6.

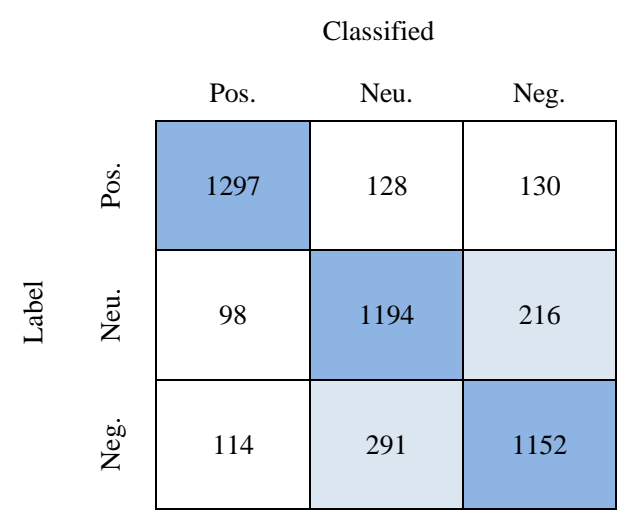

Fig. 6. Diagram of the confusion matrix corresponding to the random forests emotion recognition model.

In confusion matrix, the vertical direction expresses the emotion labelled, and the horizontal direction expresses the classified emotion. As seen in the figure, the quantity about the misclassification of that neutral classified to negative and negative classified to neutral are more than others. What lead to the conclusion maybe that the difference of stroke between individuals weaken the difference of intraindividual.

\section{Conclusion and future work}

This paper verified the possibility of that we can use the finger stroke behavior to recognize users' emotion states, and compare the difference of stroke features between male and female under different emotion states. Then we used three classified methods of machine learning including BayesNet, Back-Propagation Neutral Network and RandomForest to acquire the accuracy of emotion recognition from the perspective of individual, different gender and all subjects.

In pursuing future work, we will further collect more users' data to establish emotion recognition system on one hand. On the other hand, the emotion classified of positive, neutral and 
negative are coarse-grained, for instance, positive emotion can be divided into exciting and happy on the basis of arousal level, and we will consider the fine-grained of classified emotion in the future.

We hope our work can provide a reference to humancomputer interaction and emotion recognition in the field of artificial intelligence such as robot.

\section{References}

[1] Matthew J. Hertenstein, Dacher Keltner. Touch Communicates Distinct Emotions. Emotion, 2006, 6(3): 528-533.

[2] Matthew J. Hertenstein, Rachel Holmes, Margaret McCullough, etc. The Communication of Emotion via Touch. Emotion, 2009, 9(4): 566-573.

[3] Erin H. Thompson, James A. Hampton. The effect of relationship status on communicating emotions through touch. Cognition and Emotion, 2011, 25(2): 295-306

[4] Steve Yohanan, Karon E. MacLean. The Role of Affective Touch in Human-Robot Interaction: Human Intent and Expectations in Touching the Haptic Creature. Int J Soc Robot (2012) 4:163-180.

[5] Anna Flagg, Karon MacLean. Affective Touch Gesture Recognition for a Furry Zoomorphic Machine. TEI 2013, Feb 10-13, 2013: 25-32.

[6] Zheng Lin, Xiaolong Jin, Xueqi Cheng, etc. Make It Possible: Multilingual Sentiment Analysis without Much Prior Knowledge. 2014 IEEE/ WIC/ ACM International Joint Conferences on Web Intelligence (WI) and Intelligent Agent Technologies (IAT): 79-86.

[7] Zheng Lin, Songbo Tan, XueQi. Language-independent Sentiment Classification Using Three Common Words. CIKM'11, October 24-28,
2011: 1041-1045.

[8] Shenghua Liu, Wenjun Zhu, Xue-qi Cheng, etc. Co-training and Visualizing Sentiment Evolvement for Tweet Events. WWW 2013 Companion, May 13-17, 2013:105-106.

[9] Shenghua Liu, Fuxin Li, Xueqi Cheng, etc. Adaptive Co-Training SVM for Sentiment Classification on Tweets. CIKM'13, Oct. 27-Nov. 1, 2013 : 2079-2088.

[10] Eun-Hye Jang, Byoung-Jun Park, Sang-Hyeob Kim, etc. Emotion Classification based on Bio-Signals Emotion Recognition using Machine Learning Algorithms. Information Science, Electronics and Electrical Engineering (ISEEE), 2014:1373-1376.

[11] Wei-Long Zheng, Bo-Nan Dong, Bao-Liang Lu. Multimodal Emotion Recognition using EEG and Eye Tracking Data. Engineering in Medicine and Biology Society (EMBC), 2014 36th Annual International Conference of the IEEE, 2014: 5040- 5043.

[12] Preeti Khanna, M.Sasikumar. Recognising Emotions from Keyboard Stroke Pattern. International Journal of Computer Applications (09758887), Volume 11-No.9, December 2010: 1-5.

[13] Anna Flagg, Diane Tam, Karon MacLean, etc. Conductive Fur Sensing for a Gesture-Aware Furry Robot. IEEE Haptics Symposium, 2012: 99104.

[14] Merel M. Jung, Ronald Poppe, Mannes Poel, etc. Touching the Void Introducing CoST: Corpus of Social Touch. ICMI'14, November 12-16, 2014: 120-127.

[15] YUAN GAO, NADIA BIANCHI-BERTHOUZE, HONGYING MENG What does touch tell us about emotions in touchscreen-based gameplay? TOCHI, Nov. 2012: 39-71.

[16] Gerald Bauer, Paul Lukowicz. Can Smartphones Detect Stress-Related Changes in the Behaviour of Individuals? Work in Progress session at PerCom 2012: 423-426. 